\title{
LoCuSS: comparison of observed X-ray and lensing galaxy cluster scaling relations with simulations (Corrigendum)
}

\author{
Y.-Y. Zhang ${ }^{1,2}$, A. Finoguenov ${ }^{1,3}$, H. Böhringer ${ }^{1}$, J.-P. Kneib ${ }^{4}$, G. P. Smith ${ }^{5}$, R. Kneissl ${ }^{6}$, N. Okabe ${ }^{7}$, and H. Dahle ${ }^{4}$
}

1 Max-Planck-Institut für extraterrestrische Physik, Giessenbachstraße, 85748 Garching, Germany e-mail: yyzhang@astro.uni-bonn.de

2 Argelander-Institut für Astronomie, Universität Bonn, Auf dem Hügel 71, 53121 Bonn, Germany

3 University of Maryland, Baltimore County, 1000 Hilltop Circle, Baltimore, MD 21250, USA

4 OAMP, Laboratoire d'Astrophysique de Marseille, traverse du Siphon, 13012 Marseille, France

5 School of Physics and Astronomy, University of Birmingham, Edgbaston, Birmingham, B152TT, UK

6 Max-Planck-Institut für Radioastronomie, Auf dem Hügel 69, 53121 Bonn, Germany

7 Astronomical institute, Tohoku University, Aramaki, Aoba-ku, Sendai, 980-8578, Japan

A\&A 482, 451-472 (2008), DOI: 10.1051/0004-6361:20079103

\section{ABSTRACT}

The article by Zhang et al. (2008, A\&A, 482, 451) contains an error in Table A.1 in online material (p. 3). In preparing the final version of Table A.1, we inadvertently used the wrong conversion from degree to hh:mm:ss, with the result that the cluster coordinates were not correctly tabulated in Table A.1. We note that this does not affect any results in Zhang et al. (2008). Nevertheless, we regret this error and provide the actual cluster coordinates used in our analysis in Table A.1 in the following page.

Key words. cosmology: observations - galaxies: clusters: general - X-rays: galaxies: clusters - dark matter - gravitational lensing errata, addenda

Acknowledgements. The authors would like to thank Bradford Benson and Marion Schmitz for pointing out this error in the manuscript.

\section{References}

Zhang, Y.-Y., Finoguenov, A., Böhringer, H., et al. 2008, A\&A, 482, 451 


\section{Appendix A: X-ray mass modeling}

Table A.1.

\begin{tabular}{|c|c|c|c|c|c|c|c|c|c|c|}
\hline \multirow{2}{*}{ Name } & \multicolumn{2}{|c|}{ X-ray centroid } & \multirow[t]{2}{*}{ Id } & \multirow[t]{2}{*}{$\overline{\text { Date }}$} & \multicolumn{2}{|c|}{ Filter } & \multirow{2}{*}{$\begin{array}{c}\text { Frame } \\
\text { pn }\end{array}$} & \multicolumn{3}{|c|}{ Net exposure (ks) } \\
\hline & RA & Dec & & & MOS & pn & & MOS1 & MOS2 & pn \\
\hline RXC J0043.4-2037 & $00: 43: 24.5$ & $-20: 37: 31.2$ & 0042340201 & $2002-01-04$ & Thin & Thin & EFF & 11.4 & 11.4 & 7.1 \\
\hline RXC J0232.2-4420 & $02: 32: 18.8$ & $-44: 20: 51.9$ & 0042340301 & 2002-07-11 & Thin & Thin & EFF & 12.7 & 12.1 & 8.7 \\
\hline RXC J0307.0-2840 & $03: 07: 02.2$ & $-28: 39: 55.2$ & 0042340501 & 2001-02-16 & Thin & Thin & EFF & 12.4 & 12.7 & 9.3 \\
\hline RXC J0516.7-5430 & $05: 16: 35.2$ & $-54: 30: 36.8$ & 0205330301 & 2004-01-13 & Thin & Thin & FF & 10.6 & 11.1 & 8.9 \\
\hline RXC J0528.9-3927 & $05: 28: 52.5$ & $-39: 28: 16.7$ & 0042340801 & 2001-09-15 & Thin & Thin & EFF & 7.2 & 6.9 & 3.7 \\
\hline RXC J0532.9-3701 & $05: 32: 55.9$ & $-37: 01: 34.5$ & 0042341801 & 2002-10-07 & Thin & Thin & EFF & 10.5 & 11.3 & 7.3 \\
\hline RXC J0547.6-3152 & $05: 47: 38.3$ & $-31: 52: 28.8$ & 0201900901 & 2004-03-07 & Thin & Thin & EFF & 21.8 & 22.1 & 17.6 \\
\hline RXC J0645.4-5413 & $06: 45: 30.0$ & $-54: 13: 42.1$ & 0201901201 & 2004-05-07 & Thin & Thin & EFF & 11.6 & 12.2 & 9.6 \\
\hline RXC J0658.5-5556 & $06: 58: 30.2$ & $-55: 56: 33.7$ & 0112980201 & $2000-10-21$ & Thin & Thin & EFF & 25.7 & 23.7 & 21.1 \\
\hline RXC J0945.4-0839 & $09: 45: 25.1$ & $-08: 39: 11.7$ & 0017540101 & 2001-12-02 & Medium & Medium & FF & 7.1 & 6.4 & 4.4 \\
\hline RXC J0958.3-1103 & $09: 58: 21.9$ & $-11: 03: 48.2$ & 0201903501 & 2004-06-17 & Thin & Thin & EFF & 7.9 & 7.9 & 5.3 \\
\hline RXC J2129.6+0005 & $21: 29: 39.8$ & $+00: 05: 18.5$ & 0093030201 & $2002-10-29$ & Medium & Medium & EFF & 38.4 & 39.4 & 25.6 \\
\hline RXC J2218.6-3853 & $22: 18: 39.9$ & $-38: 53: 43.6$ & 0201903001 & 2004-10-24 & Thin & Thin & $\mathrm{EFF}$ & 20.4 & 20.3 & 12.0 \\
\hline RXC J2234.5-3744 & $22: 34: 27.1$ & $-37: 44: 07.5$ & 0018741701 & 2001-05-03 & Thin & Thin & FF & 6.7 & 6.6 & 4.2 \\
\hline RXC J2308.3-0211 & $23: 08: 22.3$ & $-02: 11: 32.1$ & 0205330501 & 2004-06-05 & Thin & Thin & $\mathrm{FF}$ & 9.6 & 10.0 & 7.8 \\
\hline RXC J2337.6+0016 & $23: 37: 37.8$ & $+00: 16: 15.5$ & 0042341301 & 2001-12-06 & Thin & Thin & EFF & 12.4 & 12.0 & 8.3 \\
\hline Abell68 & $00: 37: 06.2$ & $+09: 09: 28.7$ & 0084230201 & 2001-12-14 & Medium & Medium & EFF & 24.9 & 23.8 & 18.2 \\
\hline Abell115 & $00: 55: 50.1$ & $+26: 24: 35.7$ & 0203220101 & 2004-07-16 & Medium & Medium & EFF & 36.0 & 36.8 & 29.5 \\
\hline Abell209 & $01: 31: 52.6$ & $-13: 36: 35.5$ & 0084230301 & 2001-01-15 & Medium & Medium & EFF & 17.3 & 6.1 & 12.8 \\
\hline Abell267 & $01: 52: 42.0$ & $+01: 00: 41.2$ & 0084230401 & 2002-01-02 & Medium & Medium & EFF & 17.2 & 17.1 & 12.4 \\
\hline Abell383 & $02: 48: 03.3$ & $-03: 31: 43.6$ & 0084230501 & $2002-08-17$ & Medium & Medium & EFF & 28.1 & 28.0 & 21.5 \\
\hline Abell773 & $09: 17: 52.9$ & $+51: 43: 19.4$ & 0084230601 & 2001-04-26 & Medium & Medium & EFF & 13.0 & 14.7 & 15.9 \\
\hline Abell781 & $09: 20: 24.8$ & $+30: 30: 05.7$ & 0150620201 & 2003-04-22 & Medium & Medium & FF & 14.0 & 14.1 & 11.6 \\
\hline Abell901 & $09: 55: 57.7$ & $-09: 59: 06.3$ & 0148170101 & 2003-05-06 & Thin & Thin & $\mathrm{FF}$ & 19.4 & 18.6 & 53.2 \\
\hline Abell963 & $10: 17: 03.2$ & $+39: 02: 56.5$ & 0084230701 & 2001-11-02 & Medium & Medium & EFF & 23.8 & 24.7 & 17.9 \\
\hline Abell1413 & $11: 55: 18.3$ & $+23: 24: 12.7$ & 0112230501 & $2000-12-06$ & Thin & Thin & $\mathrm{FF}$ & 23.6 & 24.4 & 18.8 \\
\hline Abell1689 & $13: 11: 29.3$ & $-01: 20: 26.7$ & 0093030101 & 2001-12-24 & Thin & Thin & EFF & 36.8 & 37.0 & 32.6 \\
\hline Abell1758 & $13: 32: 44.6$ & $+50: 32: 46.5$ & 0142860201 & 2002-11-12 & Medium & Medium & FF & 38.1 & 40.1 & 19.4 \\
\hline Abell1763 & $13: 35: 18.1$ & $+41: 00: 03.9$ & 0084230901 & $2002-12-13$ & Medium & Medium & EFF & 12.3 & 12.0 & 9.3 \\
\hline Abell1835 & $14: 01: 01.9$ & $+02: 52: 35.5$ & 0098010101 & $2000-06-28$ & Thin & Thin & FF & 25.3 & 25.3 & 24.7 \\
\hline Abell1914 & $14: 26: 00.8$ & $+37: 49: 38.8$ & 0112230201 & 2002-12-18 & Thin & Medium & EFF & 20.5 & 21.2 & 14.5 \\
\hline Abell2204 & $16: 32: 47.1$ & $+05: 34: 32.3$ & 0112230301 & 2001-09-12 & Medium & Medium & FF & 17.5 & 18.5 & 14.3 \\
\hline Abell2218 & $16: 35: 53.8$ & $+66: 12: 32.4$ & 0112980101 & $2002-09-28$ & Thin & Thin & EFF & 16.7 & 16.9 & 13.8 \\
\hline Abell2261 & $17: 22: 26.0$ & $+32: 07: 47.4$ & 0093031001 & 2003-08-29 & Thin & Thin & EFF & 2.7 & 2.9 & 0.9 \\
\hline Abell2390 & $21: 53: 37.1$ & $+17: 41: 46.4$ & 0111270101 & 2001-06-19 & Thin & Thin & FF & 10.3 & 10.0 & 8.8 \\
\hline Abell2667 & $23: 51: 39.2$ & $-26: 05: 03.5$ & 0148990101 & 2003-06-21 & Medium & Medium & FF & 22.2 & 22.9 & 14.1 \\
\hline Z7160 & $14: 57: 15.2$ & $+22: 20: 31.2$ & 0108670201 & 2002-08-03 & Medium & Medium & FF & 31.2 & 31.6 & 26.3 \\
\hline
\end{tabular}

Notes. The cluster center is in sky coordinates in epoch J2000. The MOS data are in EE mode except that the MOS1 data of Abell1835 are in window mode, which cannot be used for this work. 